\title{
Comparative evaluation of blood salvage techniques in patients undergoing cardiac surgery with cardiopulmonary bypass
}

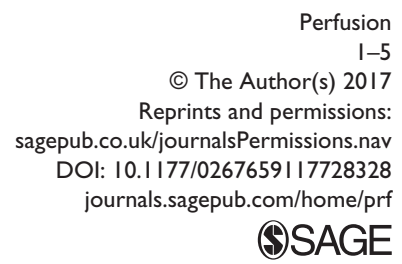

(SAGE

\author{
Serdar Gunaydin,' Craig Robertson, ${ }^{2}$ Ali Baran Budak' \\ and Terence Gourlay ${ }^{2}$
}

\begin{abstract}
Background: The primary objective of this study was to test and compare the efficacy of currently available intraoperative blood salvage systems via a demonstration of the level of increase in percentage concentration of red blood cells (RBC), white blood cells $9 \mathrm{WBC}$ ) and platelets (PIt) in the end product.

Methods: In a prospective, randomized study, data of 80 patients undergoing elective cardiac surgery with cardiopulmonary bypass in a 6-month period was collected, of which the volume aspirated from the surgical field was processed by either the HemoSep Novel Collection Bag (Advancis Surgical, Kirkby-in-Ashfield, Notts, UK) (N=40) (Group I) or a cell- saver (C.A.T.S Plus Autotransfusion System, Fresenius Kabi, Bad Homburg, Germany) (N=40) (Group 2).

Results: Hematocrit levels increased from $23.05 \% \pm 2.7$ to $43.02 \% \pm 12$ in Group I and from $24.5 \pm 2$ up to $55.2 \pm 9$ in Group $2(p=0.013)$. The mean number of platelets rose to $225200 \pm 47000$ from II $6400 \pm 40000$ in the HemoSep and decreased from $125200 \pm 25000$ to $96500 \pm 30000$ in the cell-saver group $(p=0.0000 \mathrm{I})$. The leukocyte count was concentrated

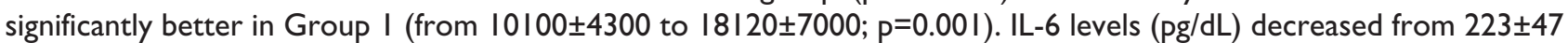
to $83 \pm 2$ I in Group I and from $219 \pm 40$ to $200 \pm 40$ in Group 2 ( $p=0.00$ I). Fibrinogen was protected significantly better in the HemoSep group (from $185 \pm 35$ to $455 \pm 45 ; \mathrm{p}=0.004$ ).

Conclusions: Intraoperative blood salvage systems functioned properly and the resultant blood product was superior in terms of red blood cell species. The HemoSep group had significantly better platelet and leukocyte concentrations and fibrinogen content.
\end{abstract}

\section{Keywords}

blood; ultrafiltration; cell-saver; cardiopulmonary bypass

\section{Introduction}

Despite the recent introduction of a number of technical and pharmacologic blood conservation measures, bleeding and allogeneic transfusion remain persistent problems in cardiac surgical procedures. The use of blood products carries several risks, such as immunologic sensitization, anaphylactic reaction and disease transmission. ${ }^{1}$ Efforts should be made to decrease or completely avoid transfusions to avoid these negative reactions. The World Health Organization encourages all member states to implement Patient Blood Management programs, employing multiple combined strategies to increase and preserve autologous erythrocyte volume in order to minimize unnecessary exposure to RBC transfusions. ${ }^{2}$

Direct cardiotomy suction from the surgical field and unprocessed blood re-transfusion are common practices during cardiopulmonary bypass $(\mathrm{CPB})$, but which are associated with a powerful activation of the coagulation and inflammatory systems: thrombin generation, excessive fibrinolysis and the release of proinflammatory cytokines. Compared with direct cardiotomy suction, the use of RBC salvage technology is

\footnotetext{
'Department of Cardiovascular Surgery, Numune Training \& Research Hospital, Ankara, Turkey

${ }^{2}$ Department of Biomedical Engineering, University of Strathclyde, Glasgow, UK

Corresponding author:

Serdar Gunaydin, Director of Cardiovascular Surgery, Numune Training \& Research Hospital, University of Health Sciences, Ankara, 06836, Turkey.

Email: sgunaydin@isnet.net.tr
} 
able to reduce the amount of microparticles and activated proteins in autologous blood before re-transfusion. Clinical studies are discordant regarding the benefit of RBC salvage use during and after cardiac operations. $^{3}$

Ultrafiltration devices remove unwanted excess plasma water, solutes, platelet inhibitors, some particulate matter and other waste substances through hemoconcentration. A number of technologies have evolved to fulfill this task over the past decades. ${ }^{4-6}$

The HemoSep ${ }^{\mathrm{TM}}$ and autotransfusion (C.A.T.S Plus) systems are commonly used devices during major operations to improve hemoglobin levels and to reduce blood product requirements with no impairment on blood coagulation.

The primary objective of this study was to observe and compare the efficacy of currently available intraoperative blood salvage systems via a demonstration of the level of increase in percentage concentration of RBCs, WBCs and Plt in the end product.

\section{Methods}

\section{Patients}

After institutional review board approval (14-16/227-21) in a tertiary cardiovascular center, 80 patients undergoing cardiac surgery with cardiopulmonary bypass in a 6-month period were prospectively randomized in that volume aspirated from the surgical field was processed by either HemoSep Novel Collection ( $\mathrm{N}=40)$ (Group 1) or cell- saver ( $\mathrm{N}=40)$ (Group 2).

Patients were randomly allocated by envelope technique in two operating rooms, each using HemoSep and cell saver technology. Patient data was recorded, including parameters within the routine blood salvage quality assessment protocol of the hospital blood management council. Only salvaged blood was evaluated. No transfusion or clinical outcome data were included.

Patients aged 18 years or over, having given his/her written informed consent, were included in the study. Patients under the age of 18, participating in another therapeutic trial or pregnant were excluded.

\section{HemoSep Novel Ultrafiltration System}

The HemoSep system consists of three major components: the HemoSep bag, a fixed rate orbital shaker and a transfer pack for the collection of processed blood. The bag element of the system is the active processing section of the device. It consists of a polyvinyl cellulose (PVC) blood bag with a polycarbonate membrane bag suspended within it, which is a sheet of super-adsorber material. The super-adsorber element is the driving force behind the transport of fluid (plasma) from the blood pool, through the control membrane and into the super-adsorber section. The key to the HemoSep device is its simplicity. Rather than moving water, it actually removes plasma. It should not spare proteins. In this regard, it is very similar to centrifuge technology, with the key difference that it spares all cell species. The priming process ensures an initial intimate contact between the fluid component of the blood product and the wetted, activated, super-adsorber. This creates channels for the fluid to be drawn into the super-adsorber material via the controlling pores. The mobility of the super-adsorber component of the HemoSep device is restricted by two factors: firstly, the gelling property of the material itself and secondly, the presence of the containing control membrane. One gram of super-adsorber will adsorb up to $240 \mathrm{ml} / \mathrm{g}$ of fluid before reaching saturation; the $12 \mathrm{gm}$ of super-adsorber employed in the device is, therefore, capable of removing up to $3 \mathrm{~L}$ of fluid before becoming saturated. Although the HemoSep device is capable of concentrating blood cells passively, the time to achieve the desired concentration is considerably shortened by employing some agitation of the device. A fixed rate of 120 cycles per minute has been determined as the most efficient frequency for the system, leading to maximal improvement in the exchange performance with no impact upon cellular damage.

The device consists of a PVC blood bag $12 \times 7$ inches in size which is flat-packed when presented for use. This processing bag, which is the main functional component of the device, weighs less than $100 \mathrm{~g}$.

Before introducing blood into the HemoSep bag, the system was first primed using $150 \mathrm{ml}$ of normal saline solution. The bag was then gently rotated by hand to ensure that the polycarbonate control membrane was adequately wetted until the priming liquid was entirely adsorbed into the super-adsorber pad. Once the volume of blood had been introduced, the inlet and outlet tubes were clamped and the bag placed into the orbital shaker chamber. The shaker was switched on and orbital, periodic, bi-directional agitation initiated at a rate of 120 cycles per second for a period of 15 minutes. Once the pre-determined time had been attained, the HemoSep bag was removed from the orbital shaker and held in an upright position with the blood outlet port at the bottom. With the blood collection bag connected to the outlet port, the outlet clamp was released and the processed blood drained into the collection bag (Figure 1).

\section{C.A.T.S Plus Autotransfusion System}

C.A.T.S ${ }^{\circledR}$ Plus is the only autotransfusion device on the market using a continuous blood flow technique. The continuous flow concept is a patented technique for washing blood in various types of surgeries, which makes it possible to use one set only for all applications, 


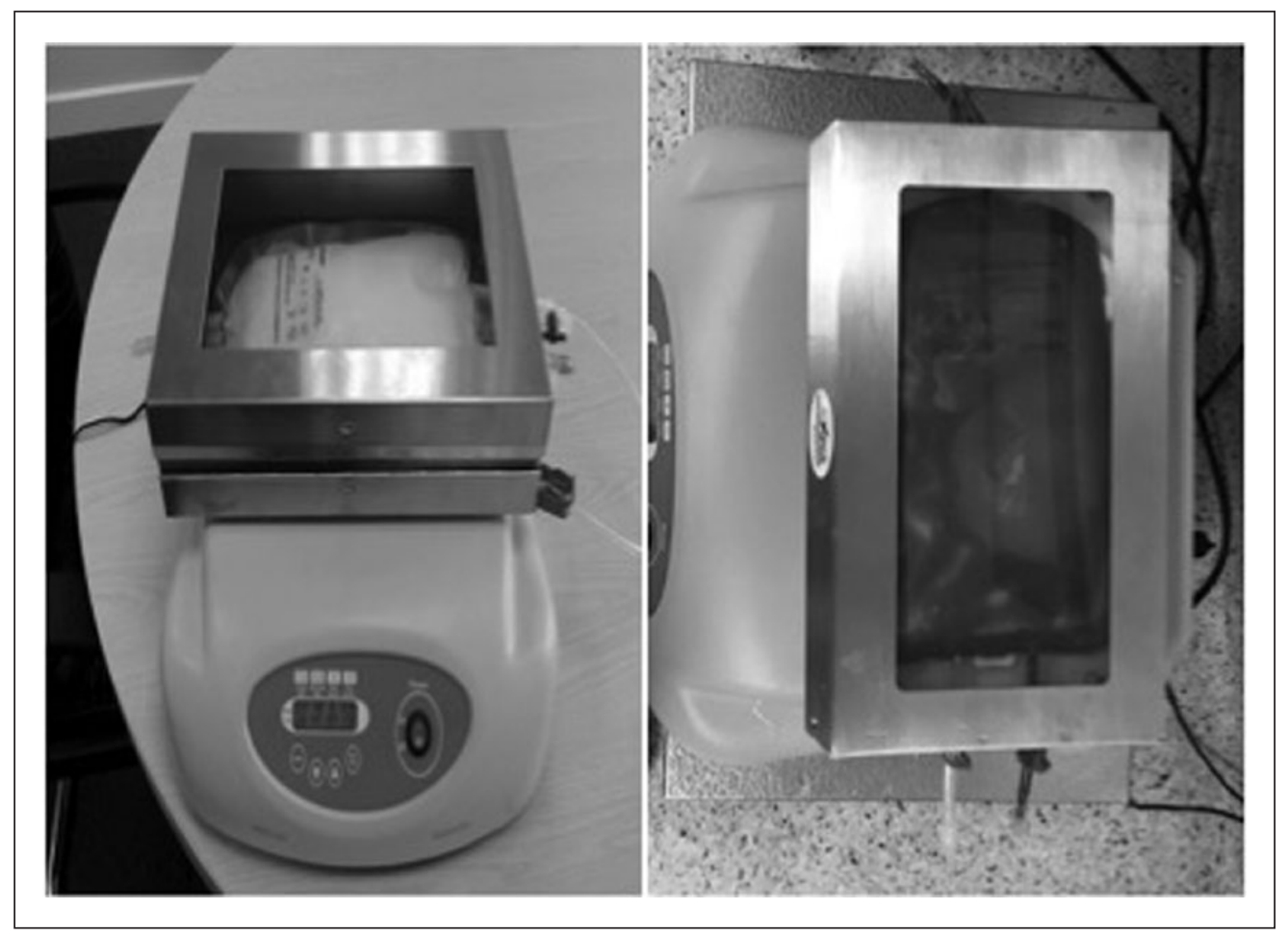

Figure I. HemoSep ultrafiltration bag on the shaker.

independent of the bleeding volumes. The continuous washing process eliminates non-emulsified fat originating in bone marrow or subcutaneous tissue. The fully automatic procedure allows separating patient blood into packed red cells, platelet rich plasma and platelet poor plasma. The emergency wash program produces up to $100 \mathrm{ml}$ of packed red cells per minute (uninterrupted operation) (Figure 2).

Blood Samples and Assays: Samples were obtained from the end-product via a port in the bags into potassium-EDTA tubes from processed blood at the baseline and $15^{\text {th }}$ min to document hematologic parameters and IL-6. Complete blood count, activated clotting time (ACT) and fibrinogen levels were evaluated. Standard blood biochemistry, especially albumin fraction, was documented. Serum interleukin 6 (IL-6) levels were measured by ELISA (Bender Medsystems, Vienna; $\mathrm{CV}<10 \%$, sensitivity $<1.4 \mathrm{pg} / \mathrm{ml}$ ). Alpha-angle, $k$-time and maximum amplitude were measured by thromboelastography (ROTEM).

Statistical Analysis: Data were expressed as the mean \pm the standard error of the mean. The Mann Whitney-U test was used to compare demographic and non-parametric data. Two-way analysis of variance (ANOVA), with factor group and repeated factor time, was used to analyze differences over time in each group and for differences between groups. A post hoc test (Bonferroni correction) was applied whenever a significant difference was detected. A p-value less than 0.05

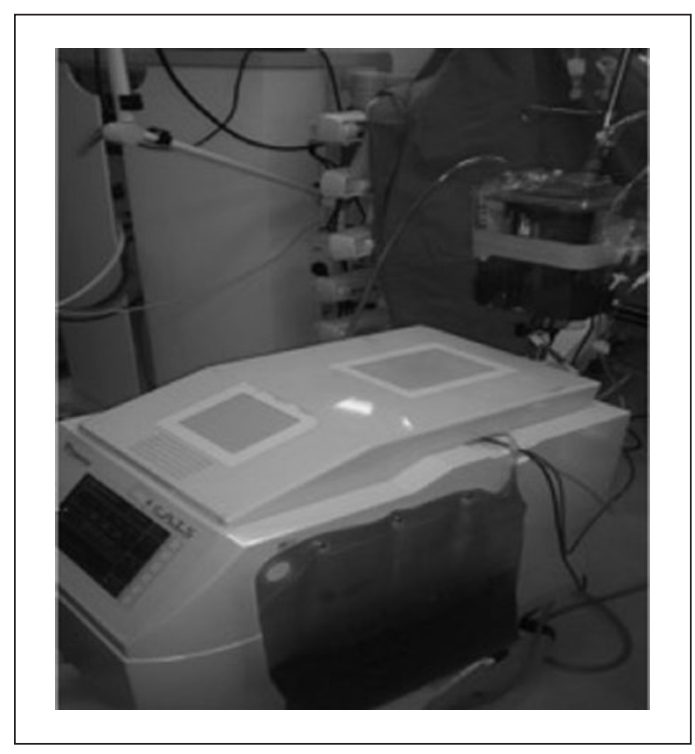

Figure 2. C.A.T.S cell saver device with collection and waste bags.

was considered significant. Data were analyzed using an SPSS program.

\section{Results}

Demographic data of both groups was comparable (27 male in the HemoSep and 24 in the cell-saver groups, 
Table I. Concentration of blood elements.

\begin{tabular}{|c|c|c|c|c|c|c|c|}
\hline & \multicolumn{2}{|l|}{ HemoSep } & \multirow[t]{2}{*}{$P$} & \multicolumn{2}{|l|}{ C.A.T.S } & \multirow[t]{2}{*}{$\mathrm{P}$} & \multirow[t]{2}{*}{ P (Compared) } \\
\hline & Baseline & 15. $\min$ & & Baseline & 15. $\min$ & & \\
\hline Hemoglobin (g/dL) & $7.68 \pm 0.9$ & $14.36 \pm 4$ & 0.0027 & $7.87 \pm 0.6$ & $18.4 \pm 3$ & 0.001 & 0.013 \\
\hline Hematocrit (\%) & $23.05 \pm 2.7$ & $43.02 \pm 12$ & 0.0026 & $24.5 \pm 2$ & $55.2 \pm 9$ & 0.001 & 0.019 \\
\hline Platelet (nX1000) & $1 \mid 6.4 \pm 40$ & $225.2 \pm 47$ & 0.0028 & $125.2 \pm 25$ & $96.5 \pm 30$ & 0.719 & 0.00001 \\
\hline White Blood Cells (n) & $10.01 \pm 4.3$ & $18.12 \pm 7$ & 0.0027 & $9.6 \pm 5$ & $11.7 \pm 6$ & 0.039 & 0.001 \\
\hline
\end{tabular}

Table 2. Impact of devices on protein preservation and IL-6.

\begin{tabular}{|c|c|c|c|c|c|c|c|}
\hline & \multicolumn{2}{|l|}{ HemoSep } & \multirow[t]{2}{*}{$\mathrm{P}$} & \multicolumn{2}{|l|}{ C.A.T.S } & \multirow[t]{2}{*}{$P$} & \multirow[t]{2}{*}{ P (Compared) } \\
\hline & Baseline & I5. $\min$ & & Baseline & 15. $\min$ & & \\
\hline $\begin{array}{l}\text { Serum Albumin } \\
\text { (g/dL) }\end{array}$ & $1.45 \pm 0.07$ & $4.36 \pm 0.08$ & 0.0026 & $1.54 \pm 0.06$ & $1.61 \pm 0.07$ & 0.32 & 0.0045 \\
\hline $\begin{array}{l}\text { Fibrinogen } \\
\text { (mg/dL) }\end{array}$ & $185 \pm 35$ & $455 \pm 45$ & 0.008 & $179 \pm 40$ & $193 \pm 40$ & 0.18 & 0.004 \\
\hline $\begin{array}{l}\text { Serum IL-6 } \\
(\mathrm{pg} / \mathrm{dL})\end{array}$ & $223 \pm 47$ & $83 \pm 21$ & 0.0038 & $219 \pm 45$ & $200 \pm 40$ & 0.22 & 0.001 \\
\hline ACT (sec) & $432 \pm 18$ & $395 \pm 20$ & 0.73 & $441 \pm 22$ & $400 \pm 23$ & 0.61 & 0.22 \\
\hline
\end{tabular}

ACT: activated clotting time.

Table 3. Platelet function of the end-product.

\begin{tabular}{|c|c|c|c|c|c|c|c|c|c|c|c|}
\hline & $\begin{array}{l}\text { Maximun } \\
\text { Amplitud }\end{array}$ & fe $(m m)$ & $P$ & $\begin{array}{l}\text { P } \\
\text { (Compared) }\end{array}$ & $\alpha$-angle & $P$ & $\begin{array}{l}\text { P } \\
\text { (Compared) }\end{array}$ & $\begin{array}{l}\text { k-time } \\
\text { (min) }\end{array}$ & & \multirow[t]{2}{*}{$P$} & \multirow[t]{2}{*}{$\begin{array}{l}\text { P } \\
\text { (Compared) }\end{array}$} \\
\hline & Baseline & I5. $\min$ & & & Baseline $15 . \mathrm{min}$ & & & Baseline & 15. $\min$ & & \\
\hline HemoSep & $49.2 \pm 11$ & $54.7 \pm 12$ & 0.08 & 0.11 & $46.4 \pm 1|55.3 \pm 1|$ & 0.07 & 0.064 & $4.1 \pm 2.1$ & $3.6 \pm 1.9$ & 0.077 & 0.054 \\
\hline C.A.T.S & $44.2 \pm 11$ & $59.5 \pm 12$ & 0.054 & & $43.5 \pm 1|58.1 \pm 1|$ & 0.061 & & $3.88 \pm 2.1$ & $4.2 \pm 1.9$ & 0.051 & \\
\hline
\end{tabular}

with a mean age of $49.1 \pm 8$ and $44.7 \pm 9$ ). Body surface area was $1.79 \pm 0.05$ for the HemoSep and $1.82 \pm 0.05$ for the cell-saver groups.

The mean processed blood was $355 \pm 110 \mathrm{~mL}$ for the HemoSep and $405 \pm 115$ for the cell-saver at the end of 15 min. No technical failures with either of the devices were reported.

The concentration of blood elements is documented in Table 1.

Both devices were significantly better in the concentration of hemoglobin/hematocrit and WBC levels with respect to baseline. The cell-saver device was significantly better in the concentration of hemoglobin/hematocrit vs HemoSep, but the HemoSep acted significantly better on platelet concentration.

The cell-saver device did not have any impact on albumin and fibrinogen preservation nor any effect on ACT measurements. IL-6 levels were significantly lower in the HemoSep group (Table 2). Neither device caused any changes in platelet function demonstrated by thromboelastography (Table 3).
Table 4 demonstrates the overall evaluation of both devices. Concentration of whole volume efficacy was $79.9 \%$ for the HemoSep and $82.5 \%$ for the cell-saver device. Waste product following ultrafiltration was significantly less in the HemoSep.

\section{Discussion}

Cardiac surgery patients are prone to anemia from several mechanisms: intraoperative blood loss, pre-existing anemia and hemodilution. The use of RBC salvage technology has been advocated to salvage blood lost in the operative field and to reduce the need of homologous blood transfusion.

The consequence of priming of the $\mathrm{CPB}$ circuit is that the blood at the end of the procedure has a lower red cell, platelet and white cell count when compared to their native preoperative levels. ${ }^{7,8}$

For some decades now, clinicians have employed hemoconcentration, either during the operative procedure or, more commonly, on the residual blood left in 
Table 4. Overall comparison of both devices.

\begin{tabular}{lll}
\hline & HEMOSEP & C.A.T.S \\
\hline PROCESSING TIME & $15 \mathrm{MIN}$ & $\mathrm{I}$ MIN \\
ACTION MODE & COLLECT AND PROCESS & CONTINUOUS \\
CONCETRATION EFFICACY & $79.9 \pm 5 \%$ & $82.5 \pm 7 \%$ \\
(WHOLE VOLUME/END PRODUCT) & & \\
PRIMING & $150 \mathrm{~mL}$ & $50 \mathrm{~mL}$ \\
WASTE & $20 \mathrm{mg}$ & $5000 \mathrm{~mL}$ \\
\hline
\end{tabular}

the $\mathrm{CPB}$ system at the termination of $\mathrm{CPB}$ to concentrate the blood cells prior to re-administration to the patient. This approach reduces the need for donor blood and the risks associated with it and offers the benefit of increasing the native cell populations in patients after surgery supported by CPB. ${ }^{4}$

The objective of the HemoSep development was to design a hemoconcentration technology that does not require centrifugation and associated blood transfer steps. An additional benefit of the HemoSep technology is that it produces a gelatinous waste product, essentially plasma in a gel matrix, which is safer and easier to dispose of than the large volumes of fluid associated with the more common centrifugation processes. We performed the first clinical evaluation of HemoSep technology and documented its contribution in blood salvage and less need of transfusion. ${ }^{9}$

Patient blood management aims to improve patient outcome and safety by reducing the number of unnecessary RBC transfusions and vitalizing patient-specific anemia reserves.

In the setting of cardiac surgery, options to avoid unnecessary blood loss and reduce blood transfusion are manifold. These are likely to improve safety and outcome of cardiac surgery patients while potentially reducing therapeutic costs.

The 2011 Update to the Society of Thoracic Surgeons and the Society of Cardiovascular Anesthesiologists Blood Conservation Clinical Practice Guidelines has reached the consensus suggesting that some form of salvaged blood and reinfusion are reasonable as part of a blood management program to minimize blood transfusion (Class IIa, Level of Evidence C).

Cell saver technology is a comparatively older technique to use for salvaged blood following complex surgery. We have demonstrated that the cell-saver concentrates red blood cell very well, but is not that much effective in platelet and protein preservation.

The HemoSep device functions properly and the resultant blood product was superior to that of the salvaged blood in terms of all active cell species studies, suggesting some possible clinical advantage in its deployment. Fibrinogen content is also being concentrated, which is also very important in the CPB setting.

Large scale comparative studies including the data on efficacy of transfusion of salvaged blood back to the patients are warranted for the evaluation of all currently available intraoperative blood salvage systems.

\section{Declaration of Conflicting Interests}

The authors declared no potential conflicts of interest with respect to the research, authorship, and/or publication of this article.

\section{Funding}

The authors received no financial support for the research, authorship, and/or publication of this article.

\section{References}

1. Escobar GA, Cheng AM, Moore EE, et al. Stored packed red blood cell transfusion upregulates inflammatory gene expression in circulating leukocytes. Ann Surg 2007; 246: 129-134.

2. Ferraris VA, Brown JR, Despotis GJ, et al. 2011 Update to the Society of Thoracic Surgeons and the Society of Cardiovascular Anesthesiologists Blood Conservation Clinical Practice Guidelines. Ann Thorac Surg 2011; 91: 944-982.

3. Paparella D, Whitlock R. Safety of salvaged blood and risk of coagulopathy in cardiac surgery. Semin Thromb Hemost 2016; 42: 166-171.

4. Johnson HD, Morgan MS, Utley JR, Leyand SA, NguyenDuy T, Crawley DM. Comparative analysis of recovery of cardiopulmonary bypass residual blood: cell saver vs. hemoconcentrator. J Extra Corpor Technol 1994; 26: 194-199.

5. Nakamura Y, Masuda M, Toshima Y, et al. Comparative study of cell saver and ultrafiltration nontransfusion in cardiac surgery. Ann Thorac Surg 1990; 49: 973-978.

6. Samolyk KA, Beckmann SR, Bissinger RC. A new practical technique to reduce allogeneic blood exposure and hospital costs while preserving clotting factors after cardiopulmonary bypass: the Hemobag. Perfusion 2005; 20: 343-349.

7. Zahoor M, Abbass S, Khan AA, Ahmad SA. Modified ultrafiltration: role in adult cardiac surgical haemostasis. J Ayub Med Coll Abbottabad 2007; 19: 49-54.

8. Samolyk KA, Beckmann SR, Bissinger RC. A new practical technique to reduce allogeneic blood exposure and hospital costs while preserving clotting factors after cardiopulmonary bypass: the Hemobag. Perfusion 2005; 20: 343-349.

9. Gunaydin S, Gourlay T. Novel ultrafiltration technique for blood conservation in cardiac operations. Ann Thorac Surg 2013; 95: 2148-2151. 